\title{
Glomus tumor of uncertain malignant potential within the cervical spine: a case report
}

\author{
Yuxi Li"^, Kaihui Su\#, Ming Li, Shixin Lu, Zhen Che, Yuwei Liang, Lin Huang^ \\ Department of Orthopedics, Sun Yat-sen Memorial Hospital, Sun Yat-sen University, Guangzhou, China \\ \#These authors contributed equally to this work. \\ Correspondence to: Yuwei Liang; Lin Huang. Department of Orthopedics, Sun Yat-sen Memorial Hospital, Sun Yat-sen University, 107 Yanjiang West \\ Road, Guangzhou 510120, China. Email: liangyw36@mail.sysu.edu.cn; huang15@mail.sysu.edu.cn.
}

\begin{abstract}
The case report details the first glomus tumor (GT) of uncertain malignant potential within the cervical spine. The patient had been experiencing neck pain and numbness of the left side of her body for 3 months. Magnetic resonance imaging (MRI) revealed a lesion with the dimensions $22 \mathrm{~mm} \times 11 \mathrm{~mm}$ in the left side of the intervertebral foramen and epidural of C1-5. When the patient appeared aggravating symptoms, we performed an emergency surgery to relieve the spinal cord compression resulting from the growing tumor. During the surgery, a grey-brown friable tumor was observed, and the tumor was located both outside and inside of the cervical spine. Morphological and immunohistochemical (IHC) analysis showed that the lesion was a globular tumor with uncertain malignant potential. After the surgery, the patient received adjuvant radiotherapy consisting of 58.9 Gy in 23 fractions postoperatively. The MRI at 4 months after the surgery showed a progression of the tumor, at which point the patient ceased treatment. GT of uncertain malignant potential within the cervical spine lacks specific clinical manifestations and reliable non-invasive means of examination, so its diagnosis depends on pathological biopsy and IHC examination. Surgical excision is the first treatment to relieve the symptoms of nerve compression. Further research of postoperative radiotherapy and chemotherapy is required to improve treatment options.
\end{abstract}

Keywords: Glomus tumor (GT); uncertain malignant potential; cervical spine; case report

Submitted Aug 31, 2021. Accepted for publication Nov 03, 2021.

doi: 10.21037/apm-21-2751

View this article at: https://dx.doi.org/10.21037/apm-21-2751

\section{Introduction}

Glomus tumor (GT) is a rare mesenchymal tumor deriving from modified smooth muscle cells of the glomus body, comprising about $1.6 \%$ of soft tissue tumors (1-5). The GT is commonly a small, benign tumor and typically occurs in the subungual area or dermis and rarely in the visceral organs. Clinically, most GT are benign, and malignant GT or GT of uncertain malignant potential is extremely rare $(2,3,5,6)$. Here, we report a case of GT of growing within the cervical spine of a 64-year-old Chinese female. In contrast to previous cases, this is a high cervical tumor and a malignant potential-indeterminacy tumor. This tumor also presented with marked infiltrative growth (7). By observing the pathological and IHC results after surgery, we found that the tumor consisted of cells resembling modified smooth muscle cells of the normal glomus tissue, and thus typically showed immunoreactivity for vimentin (vimentin can be used as a marker of mesenchymal origin cells), and produced large amounts of type IV collagen. In this case, positivity for vimentin indicated that the tumor was of mesenchymal origin $(3,4)$. We present the following article in accordance with the CARE reporting checklist (available at https://dx.doi.org/10.21037/apm-21-2751).

^ ORCID: Lin Huang, 0000-0002-8285-2658; Yuxi Li, 0000-0002-4470-7679. 

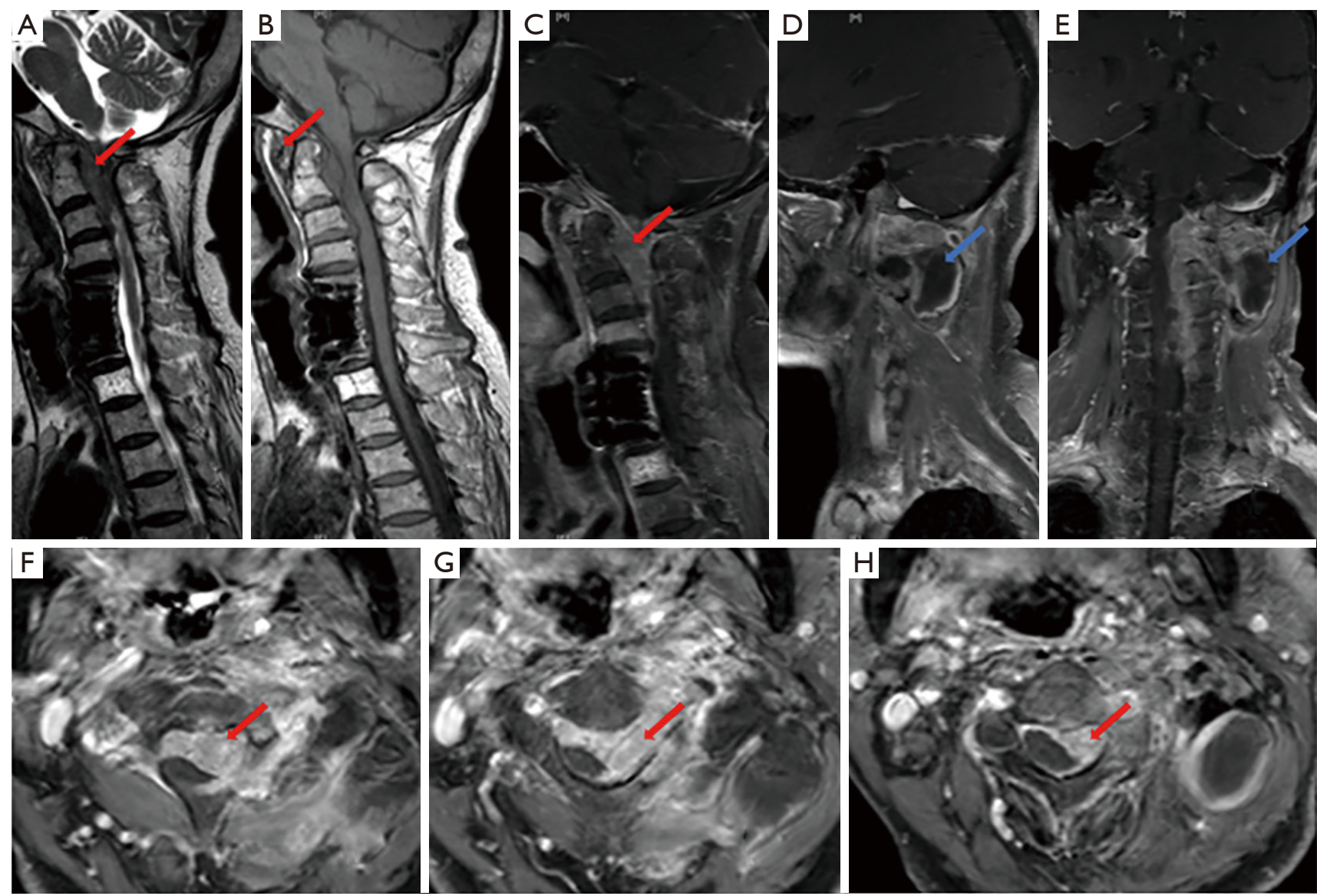

Figure $1 \mathrm{MRI}$ of the patient with an intradural extra medullary lesion. The lesion (red arrows) can be seen on T1-weighted sagittal (B) and T2-weighted sagittal (A) images, also can be well appreciated on sagittal (C) and different axial (F-H) T1 post-contrast images. In addition, on the left side of cervical vertebral side clearance cystic mass (blue arrows) is visible (D,E). Combined with the history of the disease, we suspected that it was caused by pathological puncture. MRI, magnetic resonance imaging.

\section{Case presentation}

\section{Presentation}

A 64-year-old female had been experiencing neck pain and numbness of the left side of her body for 3 months without history of accidental injury. She had received an anterior C5 corpectomy because of cervical disc herniation 3 years ago.

The plain X-ray films showed high density in the rear of C5 and C7. Computerized tomography (CT) scanning revealed an irregular poorly defined patchy lytic lesion of the left part of the occipital slope, the ring axis, the left part of the $\mathrm{C} 3$ vertebrae, and the left arch with some residual bone trabeculae remaining. Magnetic resonance imaging (MRI) (Figure 1) revealed a lesion with the dimensions $22 \mathrm{~mm} \times 11 \mathrm{~mm}$ in the left side of the intervertebral foramen and epidural space of C1-5, having slightly high signal intensity on T2-weighted imaging and equal signal intensity on T1-weighted imaging, enhancement scanning, and bright heterogeneous enhancement following gadolinium administration. There was a cystic mass with the dimensions $66 \mathrm{~mm} \times 60 \mathrm{~mm}$ on the left side of the cervical vertebral side clearance. The lesion showed low signal intensity on T2-weighted imaging, high signal intensity on T1-weighted imaging, and a ring-shaped reinforcement in enhancement scanning. All procedures performed in studies involving human participants were in accordance with the ethical standards of the institutional and/or national research committee(s) and with the Helsinki Declaration (as revised in 2013). Written informed consent was obtained from the patient for publication of this case report and accompanying images. A copy of the written consent is available for review by the editorial office of this journal. 

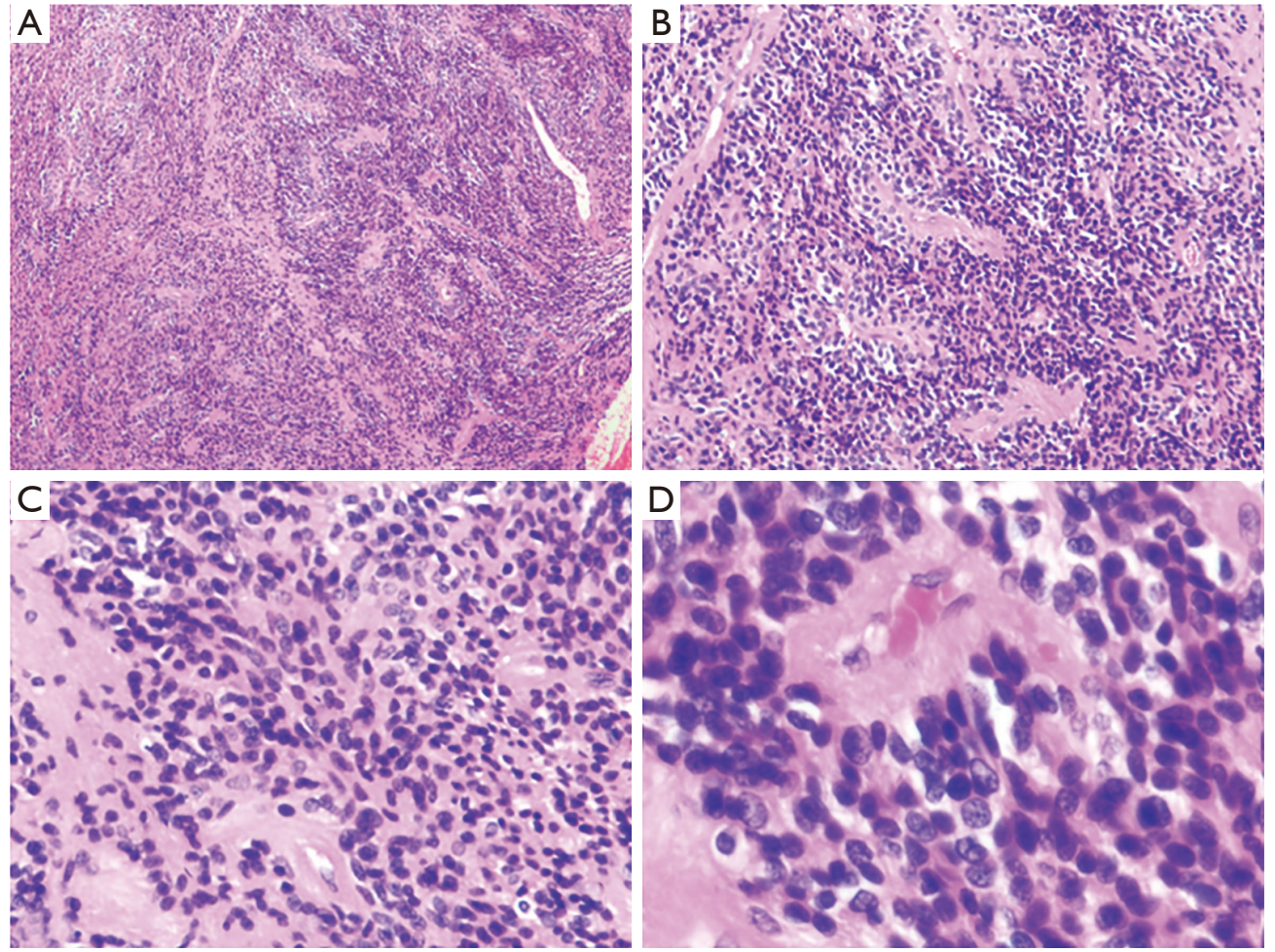

Figure $2 \mathrm{H} \& \mathrm{E}$ staining showed that the tumor was composed predominantly of sheets of ovoid to round cells. Mild-to-moderate cytological atypia was observed locally, and increased mitotic activity. (A,B: $\times 4$; C: ×10; D: ×40).

\section{Surgical procedure}

During the course of necessary examinations, the patient received regular treatment including anti-inflammatories, pain-relieving neurotrophic drugs, rehydration, and bed rest. However, the patient symptoms became aggravated, and she exhibited neurologic decline and gradually decreasing myodynamia after 2 weeks in hospital. We performed an emergency surgery to relieve the spinal cord compression resulting from the growing tumor and confirm the diagnosis. Palliative resection was performed under general anesthesia. During the surgery, a grey-brown friable tumor was observed, and the tumor was located both outside and inside of the cervical spine. Notably, in the cervical spinal canal, it was located at the ventral side of the cervical cord and extended from $\mathrm{C} 1$ to $\mathrm{C} 3$. Anterior cervical spinal cord decompression was performed and sufficient specimens were acquired. The pathological features and the imaging examination of the specimen met the criteria for skin and soft tissue of both invasive mesenchymal tumor and GT of uncertain malignancy (Figures 2,3).

\section{Follow-up}

After the surgery, the patient was transferred back to our orthopedics department, and the postoperative period was uneventful. The patient received adjuvant radiotherapy consisting of 58.9 Gy in 23 fractions postoperatively. The MRI at 4 months after the surgery showed a progression of tumor, and based on this, the patient refused further treatment including the remaining 2 radiotherapy sessions which would have completed the 70 Gy radiotherapy regime. Unfortunately, the patient was lost to follow-up and we have not been able to contact them again, which could indicate that she has passed away (Table 1).

\section{Discussion}

An extremely rare mesenchymal tumor, GT accounts for $1.6 \%$ of all soft tissue tumors. They derive from the glomus apparatus and mostly tend to grow in areas rich in glomus under the dermal reticular layer of the extremities, including fingers, toes, and under the nail bed. In rare cases, they can 

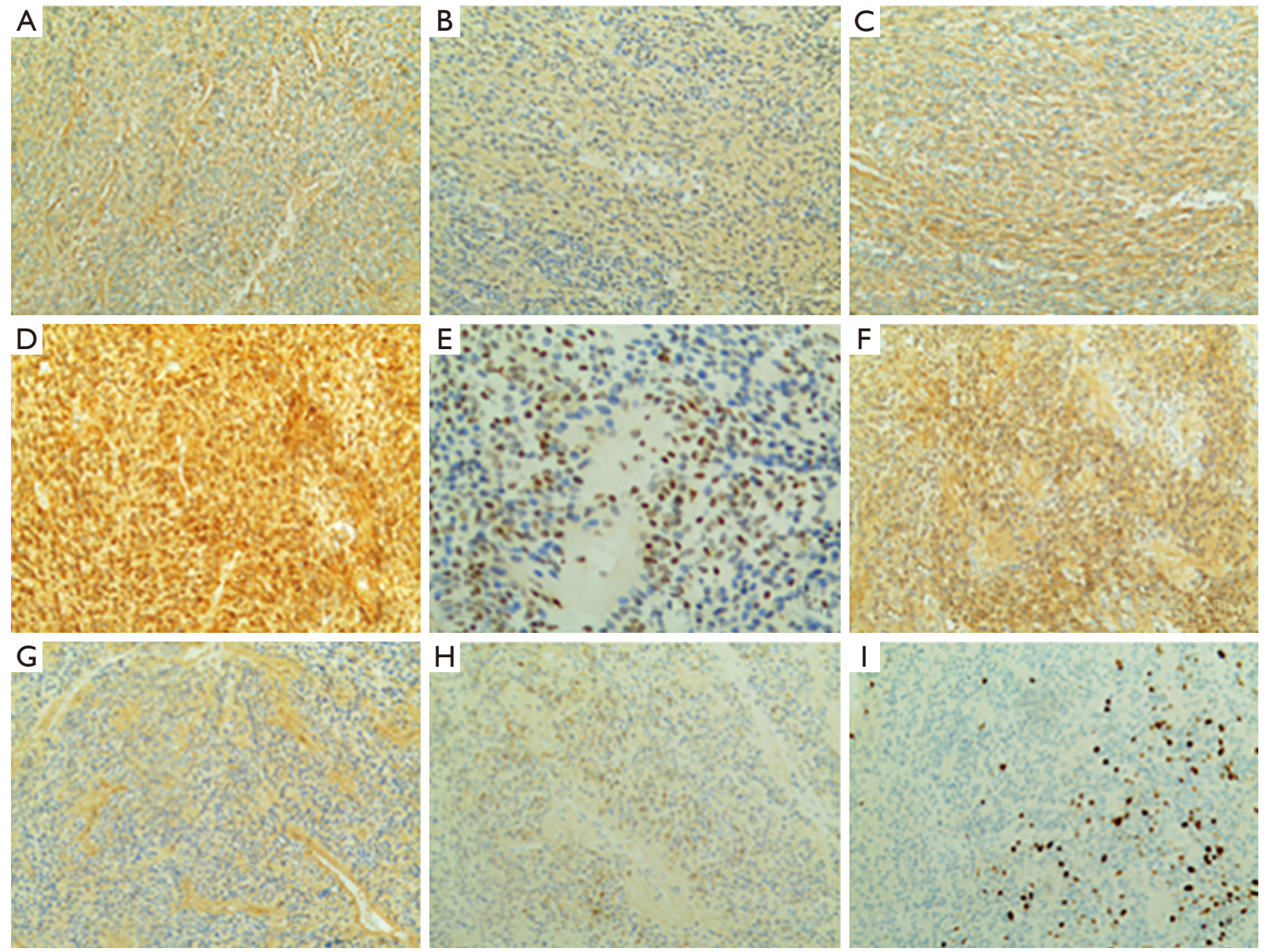

Figure 3 IHC staining showed that the cells were diffusely positive for vimentin (A), Actin (B), HHF-35 (C), and Bcl-2 (D), locally positive for Fli-1 (E), weakly positive for NSE (F) and minority of CD56, CD99 (G), Syn (H) were weakly positive. The Ki-67 proliferative index was approximately $10 \%$ (I) (A-I: ×4). IHC, immunohistochemical.

Table 1 Time-line of this patient's treatment

\begin{tabular}{|c|c|}
\hline Date & Event \\
\hline $2017-2-10$ & $\begin{array}{l}\text { Based on morphologic features and the IHC analysis, the tumor was consistent with glomus tumor of uncertain } \\
\text { malignant potential }\end{array}$ \\
\hline 2017-4-19 & The MRI at 4 months after the surgery showed a progression of the tumor \\
\hline $2017-4-21$ & $\begin{array}{l}\text { The patient gave up the treatment (the remaining } 11.1 \text { Gy of radiotherapy) and since then we have not made successful } \\
\text { contact with her }\end{array}$ \\
\hline
\end{tabular}

MRI, magnetic resonance imaging; IHC, immunohistochemical. 
occur in deep soft tissue, mediastinum, nerves, bones, or visceral organs, such as the stomach, small intestine, and lung. In addition, hemangioblastoma and paraganglioma may lead to confusion due to misuse of the terms of glomus tumor $(1,3,5,6,8-10)$. The median age of onset is 45 years (range, 28 to 79 years), and there is no significant gender difference in its incidence. Common symptoms of GT included paroxysmal pain, hypersensitivity to touch and cold; however, only about $30 \%$ of patients present these 3 typical symptoms concurrently. Examination will show subcutaneous nodules with mild tenderness. There may be positive signs for the Love's pin test and Hildreth's test in the affected area (1-3,5,6,9-11). Besides, some GT with uncommon medical manifestations such as large size, deep soft tissue or visceral location, infiltrative growth pattern, or multicentricity are classified as atypical type. If an atypical and malignant GT is in the neck, most patients only show swelling or nodules on one side of the neck and pain in the corresponding area. Invasion of adjacent organs may be associated with hoarseness, dyspnea, dysphagia, neurological dysfunction, and bone destruction $(2,10)$. For the GT within the spinal canal, patients mostly manifest as back pain, nerve root symptoms, or symptoms of spinal compression $(6,8)$. There is no significant difference from the symptoms caused by most other tumors within or near the spinal cord. Similarly, the symptoms displayed by this patient like neck pain, numbness, and weakness of the left face and left upper limb were not specifically indicative of GT. In terms of imaging examination, CT, MRI, and ultrasound all revealed signs suggesting GT; however, they all lacked signs that directly point to angioblastoma, making it difficult to diagnose clearly before surgery. Therefore, in the vast majority of cases, the diagnosis of GT relies on pathological and immunohistochemical (IHC) examination $(5,6,9,10)$.

We obtained the pathological and IHC results of this patient after surgery. Microscopically, the tumor was located inside the cervical connective tissue (Figure 2). The tumor was composed predominantly of sheets of ovoid to spherical cells with a clear border, pale cytoplasm, and fine granular chromatin which are attributes of a solid GT (1). Such cells densely surround vessels, fasciculate distribution or surrounding, which is another feature of GT. Mild-tomoderate cytological atypia was initially discovered locally, alongside increased mitotic activity [the mitotic matter was greater than 5 per 50 high-power fields (HPF)]. The tumor focally invaded the surrounding normal blood vessels. The IHC staining showed that the cells were diffusely positive for vimentin, Actin, HHF-35, and Bcl-2, locally positive for Fli-1, weakly positive for NSE, and minority of CD56, CD99, and Syn were weakly positive. The IHC staining for S-100, GFAP, EMA, Desmin, STAT-6, Melan-A, HMB45, CD34, MyoD1, Myogenin, FVIII, CD117, and DoG-1 were negative. The Ki-67 proliferative index was approximately $10 \%$. The tumor cells were surrounded by positive staining for silver. The tumor was negative for the EWSR1 gene fracture detection by fluorescence in situ hybridization (Figure 2). Folpe et al. categorized GT with nonclassical features into 4 types, which are as follows: (I) malignant GT, (II) tumors of uncertain malignant potential, (III) "symplastic" GT, and (IV) glomangiomatosis (diffuse GT) (2). According to classification of GT with nonclassical features in Enzinger and Weiss's Soft Tissue Tumors described in 2014, the diagnosis of "malignant GT" should be as followings: atypia and mitotic activity ( $>5$ per $50 \mathrm{HPF}$ ) or atypical mitotic figures, or is large $(>2 \mathrm{~cm})$ and deeply located. Most lesions falling into this class are superficial tumors with high mitotic activity and no significant nuclear atypia, or they are large or deep. However, mitotic activity of extra than 5 mitoses per $50 \mathrm{HPF}$, excessive cellularity, the presence of necrosis, and approaching moderate-to-high nuclear grade did not gain significance. The tumor consisted of cells resembling modified smooth muscle cells of the normal glomus tissue, and thus typically showed consistent immunoreactivity for SMA, MSA, and vimentin, and produced large amounts of type IV collagen. Other positive markers have been reported include but are not limited to Calponin, HHF35, VEGF, and CD34 (3,4,8,11-14). In this case, positivity for vimentin indicated that the tumor was of mesenchymal origin. Also, cytoplasmic smooth muscle markers and pericellular basement membrane constituents provided evidence for the diagnosis of GT For other common epithelioid soft tissue tumors in the neck including epithelioid hemangioma, granular cell tumor, epithelioid malignant peripheral nerve sheath tumor (MPNST), this tumor's salient histologic features and key immunophenotypes did not match up with their actual situation $(4,8,13)$. To diagnose malignant GTs, we need to consider the tumor size, infiltrative growth, growth pattern, cellularity, nuclear grade, mitotic activity, atypical mitotic figures, vascular involvement, and necrosis. World Health Organization classification of soft tissue tumors [2013] recommended that tumors with a deep location and a size of more than $2 \mathrm{~cm}$ in the absence of nuclear atypia were classified as glomus tumors of "uncertain malignant potential". Based on these criteria, the case met 
the diagnostic criteria for "GT of uncertain malignant potential (15)." In addition, the pathological features met the standards of uncertain malignant GT. Thus, we graded the tumor as 'GT of uncertain malignant potential' in spite of the unusual location in the cervical spine.

Due to the scarcity of reported cases of GT, the gold standard for its treatment has not been established in the literature. By far, complete resection is the basic treatment of GT, which is the most necessary prognostic factor $(6,11,16)$. Nevertheless, functional status should also be taken into consideration because some GT are unable to be removed completely. In particular, if the lesion extends into the spinal canal, compressing the spinal nerves and radiating pain in the lower extremities, the first task for most physicians is to relieve the spinal cord compression to preserve the spinal cord and neurofunction as soon as possible. In our case, due to the wide range and deep location of the tumor, complete resection or extended resection of significant uninvolved margins was deemed excessive. A conservative local excision might be a better option to preserve life quality and able to lessen operative morbidity.

Only a few previous articles have mentioned radiotherapy of GT. Radiotherapy of primary or metastatic GTs in the head and neck can temporarily reduce tumor volume and alleviate pain symptoms, but the control of tumor progression is unreliable $(10,17)$. Due to the positive surgical margin of the patient, it was considered that there were still soft tissue lesion residues after resection, so we chose to perform postoperative adjuvant radiotherapy. During radiotherapy, however, tumors progressed and the patient finally chose to stop treatment, meaning that we were unable to proceed with further medical interventions or evaluations. The difficulty of GT resection in the neck increases the necessity of postoperative adjuvant radiotherapy, and the optimum treatment scheme and prognosis evaluation still require further research.

\section{Conclusions}

In our previous study, in order to determine the type of tumor and the specific classification of glomus tumor, we selected a variety of common biomarkers to identify. The results showed that HHF-35, Fli-1 and CD56 were more or less expressed in glomus tumor. This is helpful for pathological diagnosis of similar cases in the future (18-20). This case report entails a rare type and location of malignant nodular tumor of the cervical spine. Data regarding GT arising in the cervical spine are currently scarce, therefore, accumulation of more cases is needed to clarify its diagnosis and significance.

\section{Acknowledgments}

We appreciate the contributions of all the surgeons involved.

Funding: This work was supported by the Guangdong Natural Science Foundation (2020A1515011388) and the Sun Yat-sen Memorial Hospital Yat-sen Qihang Youth Project (YXQH202009).

\section{Footnote}

Reporting Checklist: The authors have completed the CARE reporting checklist. Available at https://dx.doi. org/10.21037/apm-21-2751

Conflicts of Interest: All authors have completed the ICMJE uniform disclosure form (available at https://dx.doi. org/10.21037/apm-21-2751). The authors report that this work was supported by the Guangdong Natural Science Foundation (2020A1515011388) and the Sun Yatsen Memorial Hospital Yat-sen Qihang Youth Project (YXQH202009). The authors have no other conflicts of interest to declare.

Ethical Statement: The authors are accountable for all aspects of the work in ensuring that questions related to the accuracy or integrity of any part of the work are appropriately investigated and resolved. All procedures performed in studies involving human participants were in accordance with the ethical standards of the institutional and/or national research committee(s) and with the Helsinki Declaration (as revised in 2013). Written informed consent was obtained from the patient for publication of this case report and accompanying images. A copy of the written consent is available for review by the editorial office of this journal.

Open Access Statement: This is an Open Access article distributed in accordance with the Creative Commons Attribution-NonCommercial-NoDerivs 4.0 International License (CC BY-NC-ND 4.0), which permits the noncommercial replication and distribution of the article with the strict proviso that no changes or edits are made and the original work is properly cited (including links to both the 
formal publication through the relevant DOI and the license).

See: https://creativecommons.org/licenses/by-nc-nd/4.0/.

\section{References}

1. Gombos Z, Zhang PJ. Glomus tumor. Arch Pathol Lab Med 2008;132:1448-52.

2. Folpe AL, Fanburg-Smith JC, Miettinen M, et al. Atypical and malignant glomus tumors: analysis of 52 cases, with a proposal for the reclassification of glomus tumors. Am J Surg Pathol 2001;25:1-12.

3. Kurohara K, Michi Y, Yukimori A, et al. The glomus tumor resorbed bone and teeth in the mandible: a case report. Head Face Med 2018;14:18.

4. Wei S, Henderson-Jackson E, Qian X, et al. Soft Tissue Tumor Immunohistochemistry Update: Illustrative Examples of Diagnostic Pearls to Avoid Pitfalls. Arch Pathol Lab Med 2017;141:1072-91.

5. El Hyaoui H, Messoudi A, Rafai M, et al. Unusual localization of glomus tumor of the knee. Joint Bone Spine 2016;83:213-5.

6. Liu T, Zou W, Kong J, et al. Embolization in the treatment of an intraosseous glomus tumor in the upper thoracic spine complicating compression myelopathy: a case report and a literature review. Turk Neurosurg 2015;25:479-84.

7. Li X, Zheng J. Extradural glomus tumor of the thoracic spine: case report and review of the literature. Ann Palliat Med 2021;10:9309-17.

8. Oide T, Yasufuku K, Shibuya K, et al. Primary pulmonary glomus tumor of uncertain malignant potential: A case report with literature review focusing on current concepts of malignancy grade estimation. Respir Med Case Rep 2016;19:143-9.

9. Sethu C, Sethu AU. Glomus tumour. Ann R Coll Surg Engl 2016;98:e1-2.

10. Wolter NE, Adil E, Irace AL, et al. Malignant

Cite this article as: $\mathrm{Li} \mathrm{Y}$, Su K, Li M, Lu S, Che Z, Liang Y, Huang L. Glomus tumor of uncertain malignant potential within the cervical spine: a case report. Ann Palliat Med 2021;10(11):12018-12024. doi: 10.21037/apm-21-2751 glomus tumors of the head and neck in children and adults: Evaluation and management. Laryngoscope 2017;127:2873-82.

11. Zhu YZ, Li WP, Wang ZY, et al. Glomus tumor of uncertain malignant potential arising in the bronchus. J Cardiothorac Surg 2013;8:146.

12. Sansur CA, Pouratian N, Dumont AS, et al. Part II: spinalcord neoplasms--primary tumours of the bony spine and adjacent soft tissues. Lancet Oncol 2007;8:137-47.

13. Gill J, Van Vliet C. Infiltrating glomus tumor of uncertain malignant potential arising in the kidney. Hum Pathol 2010;41:145-9.

14. Wan PZ, Han Q, Wang EH, et al. Glomus tumor of uncertain malignant potential of the lung: a case report and review of literature. Int J Clin Exp Pathol 2015;8:15402-6.

15. Chen JH, Lin L, Liu KL, et al. Malignant glomus tumor of the intestinal ileum with multiorgan metastases: A case report and review of literature. World J Gastroenterol 2020;26:770-6.

16. Ropper AE, Ropper AH. Acute Spinal Cord Compression. N Engl J Med 2017;376:1358-69.

17. Horne ZD, Karam SD, Rashid A, et al. The use of stereotactic body radiation therapy for local control of glomangiomatosis: a case report. Front Oncol 2013;3:26.

18. Ariizumi $\mathrm{Y}$, Koizumi H, Hoshikawa M, et al. A primary pulmonary glomus tumor: a case report and review of the literature. Case Rep Pathol 2012;2012:782304.

19. Li Y, Luo H, Liu T, et al. The ets transcription factor Fli-1 in development, cancer and disease. Oncogene 2015;34:2022-31.

20. Fang HQ, Yang J, Zhang FF, et al. Clinicopathological features of gastric glomus tumor. World J Gastroenterol 2010;16:4616-20.

(English Language Editor: J. Jones) 